\title{
Throughput and Energy Efficiency for S-FFR in Massive MIMO Enabled Heterogeneous C-RAN
}

\author{
Anqi He*, Lifeng Wang ${ }^{\dagger}$, Yue Chen*, Kai-Kit Wong ${ }^{\dagger}$, and Maged Elkashlan* \\ * School of Electronic Engineering and Computer Science, Queen Mary University of London, London, UK \\ ${ }^{\dagger}$ Department of Electronic and Electrical Engineering, University College London, London, UK
}

\begin{abstract}
This paper considers the massive multiple-input multiple-output (MIMO) enabled heterogeneous cloud radio access network (C-RAN), in which both remote radio heads (RRHs) and massive MIMO macrocell base stations (BS) are deployed to potentially accomplish high throughput and energy efficiency (EE). In this network, the soft fractional frequency reuse (S-FFR) is employed to mitigate the inter-tier interference. We develop a tractable analytical approach to evaluate the throughput and $\mathbf{E E}$ of the entire network, which can well predict the impacts of the key system parameters such as number of macrocell BS antennas, RRH density, and S-FFR factor, etc. Our results demonstrate that massive MIMO is still a powerful tool for improving the throughput of the heterogeneous C-RAN while RRHs are capable of achieving higher EE. The impact of S-FFR on the network throughput is dependent on the density of RRHs. Furthermore, more radio resources allocated to the RRHs can greatly improve the EE of the network.
\end{abstract}

\section{INTRODUCTION}

As a new mobile network architecture consisting of remote radio heads (RRHs) and base band units (BBUs), cloud radio access network (C-RAN) can efficiently deal with largescale control/data processing. The rationale behind it is that baseband processing is centralized and coordinated among sites in the centralized BBU pool, which reduces the capital expenditure (CAPEX) and operating expenditure (OPEX) of the networks [1]. Among the emerging techniques such as device-to-device (D2D), full duplex, millimeter wave, and massive multiple-input multiple-output (MIMO), C-RAN is identified as a promising $5 \mathrm{G}$ technology $[2,3]$.

Driven by the high spectrum efficiency (SE) and energy efficiency (EE) of the C-RAN, tremendous attention has been paid from both industry and academia [3,4]. In [4], a group of single-antenna RRHs were considered to form a distributed antenna array, and two downlink transmission strategies namely best RRH selection and distributed beamforming were examined in terms of outage probability. In [5], user-centric association in a multi-tier C-RAN was proposed, where the RRH that had the best signal-to-noise ratio (SNR) was scheduled to serve the user. Compared to [4], downlink transmission in the C-RAN with a group of multi-antenna RRHs was investigated in [6].

Heterogeneous C-RAN is a new paradigm by integrating cloud computing with heterogeneous networks (HetNets) [7, 8]. In heterogeneous C-RAN, severe inter-tier interference is mitigated for the enhancement of SE and EE. In addition, massive MIMO is another essential enabling 5G technology for improving SE and EE. In massive MIMO systems, BSs equipped with large antenna arrays accommodate a large number of users in the same time-frequency domain [9]. The architecture of heterogenous C-RAN with massive MIMO is envisioned as an appealing solution, since none of these techniques can solely achieve $5 \mathrm{G}$ targets [2,7]. In [7], the opportunities and challenges for heterogenous C-RAN with massive MIMO were illustrated, in which it was mentioned that the proper densities of the massive MIMO empowered macrocell BSs and RRHs in the networks should be addressed. While the significance of heterogenous C-RAN with massive MIMO has been highlighted in the prior works [3,7], more research efforts should be devoted for comprehensively understanding it.

Although C-RAN can well mitigate the inter-RRH interference by using the efficient interference management techniques such as coordinated multi-point (CoMP), the inter-tier interference between the RRHs and macrocell BSs may be problematic in the heterogeneous C-RAN, due to the limited radio resources. The work of [8] considered soft fractional frequency reuse (S-FFR) in the heterogeneous C-RAN, and an energy-efficient resource allocation solution to jointly assign the resource block $(\mathrm{RB})$ and transmit power was obtained by using Lagrange dual decomposition.

Motivated by the aforementioned, this paper considers a two-tier heterogeneous C-RAN, where RRHs co-exit with massive MIMO aided macrocells. We focus on the EE of the $\mathrm{S}-\mathrm{FFR}$ in this network, which to the best of our knowledge, has not been conducted yet. Different from [8], we consider the spatially distributed RRHs and massive MIMO enabled macrocell BSs with the help of stochastic geometry. While the aforementioned literature $[4,6]$ considered only one single user existed in the network with multiple RRHs around the user coverage area and evaluated the performance from the standpoint of the user, we analyze the throughput and EE of the entire network by addressing the impact of tier density and massive MIMO. We first derive the exact expressions of the throughput and EE for RRHs tier. Then, we derive a closedform lower bound expression for the throughput and EE of the macrocell BSs tier. Our results show that although RRHs achieve higher EE, massive MIMO adopted by the macrocells can significantly improve the throughput of the entire network. When the number of RRHs is not dense, increasing the S-FFR factor decreases the network throughput. When a large number of RRHs is deployed, both throughput and EE of the entire network have a substantial increase. 


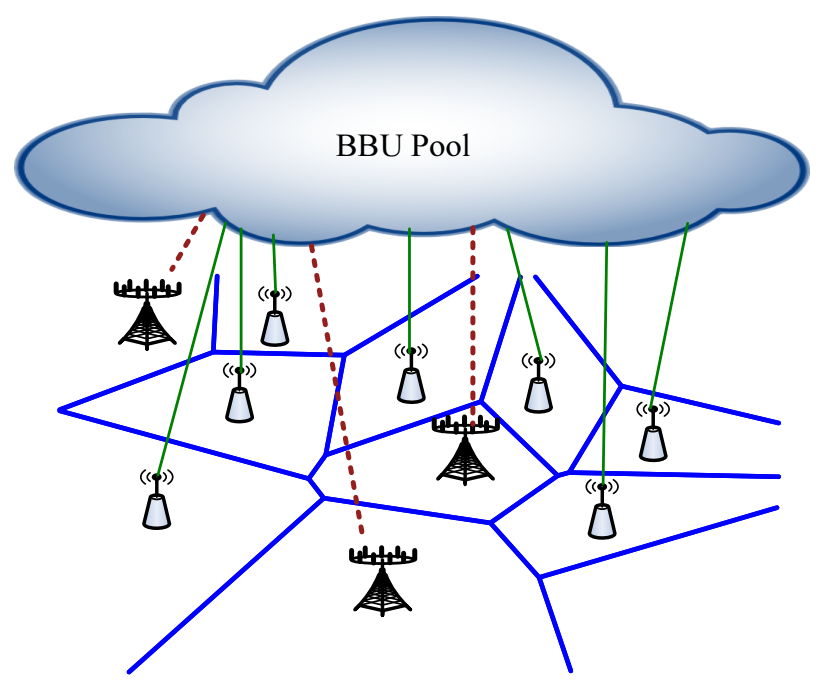

Fig. 1. An illustration of a two-tier heterogeneous C-RAN, where the red dash lines represent the backhual links between the macrocell base stations and $\mathrm{BBU}$ pool via X2/S1 interfaces, and the green solid lines represent the fronthaul links between the RRHs and BBU pool via optical fiber link.

\section{SySTEM DESCRIPTIONS}

\section{A. Network Model}

As shown in Fig. 1, we consider downlink transmission in a two-tier heterogeneous C-RAN, where the BBU pool in the cloud is established to coordinate the entire network, massive MIMO enabled macrocell BSs (MBSs) of the first tier, as high power nodes (HPNs), are connected with the BBU pool via backhaul link, and RRHs of the second tier, as low power nodes (LPNs), are connected with the BBU pool via fronthaul link (optical fiber link). The locations of MBSs are modeled following a homogeneous Poisson point process (HPPP) $\Phi_{\mathrm{M}}$ with density $\lambda_{\mathrm{M}}$, and the locations of RRHs are modeled following an independent HPPP $\Phi_{\mathrm{R}}$ with density $\lambda_{\mathrm{R}}$. Using linear zero-forcing beamforming (ZFBF), each MBS is equipped with $N_{\mathrm{M}}$ antennas and simultaneously communicates with $S$ single-antenna users over the same RB $\left(N_{\mathrm{M}} \gg S \geq 1\right)$ under equal power assignment. Each RRH is equipped with one single antenna and serves one singleantenna user over one RB. All the channels are assumed to undergo independent identically distributed (i.i.d.) quasi-static Rayleigh block fading. In this network, each user is assumed to be connected with its nearest BS such that the Euclidean plane is divided into Poisson-Voronoi cells.

We consider the S-FFR for inter-tier interference mitigation. Since the inter-RRH interference can be well mitigated and same radio resources can be shared among RRHs in the CRAN [8], the instantaneous achievable rate for a typical RRH is written as

$R_{\mathrm{RRH}}=\sum_{k=1}^{\alpha K} B_{o} \log _{2}\left(1+\gamma_{\mathrm{R}, k}\right)+\sum_{\nu=1}^{(1-\alpha) K} B_{o} \log _{2}\left(1+\gamma_{\mathrm{R}, \nu}\right)$, where

$$
\left\{\begin{aligned}
\gamma_{\mathrm{R}, k} & =\frac{P_{\mathrm{R}}}{B_{o} N_{o}} h_{\mathrm{R}, k} \beta\left|X_{o, \mathrm{R}}\right|^{-\eta_{\mathrm{R}}} \\
\gamma_{\mathrm{R}, \nu} & =\frac{P_{\mathrm{R}} h_{\mathrm{R}, \nu} \beta\left|X_{o, \mathrm{R}}\right|^{-\eta_{\mathrm{R}}}}{I_{\mathrm{M}, \nu}+B_{o} N_{o}}
\end{aligned}\right.
$$

$\alpha$ is the S-FFR factor, $K$ is the total number of RBs, $B_{o}$ is the bandwidth per RB, $P_{\mathrm{R}}$ is the RRH transmit power allocated to each $\mathrm{RB}, h_{\mathrm{R}, k} \sim \exp (1)$ and $h_{\mathrm{R}, \nu} \sim \exp (1)$ are the smallscale fading channel power gains, $\beta$ is the frequency dependent constant value, $\eta_{\mathrm{R}}$ is the pathloss exponent, $\left|X_{o, \mathrm{R}}\right|$ is the distance between the typical RRH and its intended user, and $N_{o}$ is the power spectrum density of the noise and weak inter$\mathrm{RRH}$ interference. In (1), $I_{\mathrm{M}, \nu}$ is the inter-tier interference from MBSs, which is given by

$$
I_{\mathrm{M}, \nu}=\sum_{\ell \in \Phi_{\mathrm{M}}} \frac{P_{\mathrm{M}}}{S} h_{\ell, \nu} \beta\left|X_{\ell, \mathrm{M}}\right|^{-\eta_{\mathrm{M}}},
$$

where $P_{\mathrm{M}}$ is the MBS transmit power allocated to each RB, $h_{\ell, \nu} \sim \Gamma(S, 1)$ is the small-scale fading interfering channel power gain, $\left|X_{\ell, \mathrm{M}}\right|$ is the distance between the interfering MBS $\ell \in \Phi_{\mathrm{M}}$ and the user associated with the typical RRH, and $\eta_{\mathrm{M}}$ is the pathloss exponent.

In the C-RAN, the inter-MBS interference can be coordinated by the BBU pool through backhaul link [7]. Thus, the instantaneous achievable rate for a typical MBS can be written as

$$
R_{\mathrm{MBS}}=\sum_{\nu=1}^{(1-\alpha) K} B_{o} S \log _{2}\left(1+\gamma_{\mathrm{M}, \nu}\right)
$$

where

$$
\gamma_{\mathrm{M}, \nu}=\frac{\frac{P_{\mathrm{M}}}{S} g_{\mathrm{M}, \nu} \beta\left|X_{o, \mathrm{M}}\right|^{-\eta_{\mathrm{M}}}}{I_{\mathrm{R}, \nu}+B_{o} N_{1}} .
$$

$g_{\mathrm{M}, \nu} \sim \Gamma\left(N_{\mathrm{M}}-S+1,1\right)$ is the small-scale fading channel power gain [10], $\left|X_{o, \mathrm{M}}\right|$ is the distance between the typical MBS and its intended user, $N_{1}$ is the power spectrum density of the noise and weak inter-MBS interference, and $I_{\mathrm{R}, \nu}$ is the inter-tier interference from RRHs, which is given by

$$
I_{\mathrm{R}, \nu}=\sum_{j \in \Phi_{\mathrm{R}}} P_{\mathrm{R}} g_{j, \nu} \beta\left|X_{j, \mathrm{R}}\right|^{-\eta_{\mathrm{R}}},
$$

where $g_{j, \nu} \sim \exp (1)$ and $\left|X_{j, \mathrm{R}}\right|$ are the small-scale interfering channel power gain and the distance between interfering MBS $j \in \Phi_{\mathrm{R}}$ and the user associated with the typical MBS, respectively.

\section{B. Power Consumption Model}

The total power consumption at each RRH is calculated as

$$
P_{\mathrm{R}}^{\text {total }}=K \frac{P_{\mathrm{R}}}{\varepsilon_{\mathrm{R}}}+P_{\mathrm{R}}^{0}+P_{\mathrm{fh}},
$$

where $\varepsilon_{\mathrm{R}}$ is the efficiency of the power amplifier, $P_{\mathrm{R}}^{0}$ is the static hardware power consumption of the RRH and $P_{\mathrm{fh}}$ the power consumption of the fronthaul link. 

[11]

The total power consumption at each MBS is calculated as

$$
\begin{aligned}
P_{\mathrm{M}}^{\text {total }}=(1-\alpha) & K\left(\frac{P_{\mathrm{M}}}{\varepsilon_{\mathrm{M}}}+\sum_{\rho=1}^{3}\left((S)^{\rho} \Delta_{\rho}+(S)^{\rho} N \Lambda_{\rho}\right)\right) \\
+ & P_{\mathrm{M}}^{0}+P_{\mathrm{bh}},
\end{aligned}
$$

where $\varepsilon_{M}\left(0<\varepsilon_{M} \leq 1\right)$ is the efficiency of the power amplifier, the parameters $\Delta_{\rho}$ and $\Lambda_{\rho}$ depends on the transceiver chains, coding and decoding, precoding, etc., which are detailed in the Section IV, $P_{\mathrm{M}}^{0}$ is the MBS's static hardware power consumption, and $P_{\mathrm{bh}}$ is the power consumption of the backhaul link.

\section{Performance Evaluations}

In this section, we first derive the throughput in the massive MIMO enabled heterogeneous C-RAN. Using the derived results, we evaluate the $\mathrm{EE}$ in this network.

\section{A. Throughput}

We first derive the throughput for a typical RRH, which is as follows.

Theorem 1. The throughput for a typical RRH is given by

$$
\bar{R}_{\mathrm{RRH}}=\frac{2 \pi}{\ln 2}\left(\lambda_{\mathrm{R}}+\lambda_{\mathrm{M}}\right) \int_{0}^{\infty} \phi(x) x e^{-\pi\left(\lambda_{\mathrm{R}}+\lambda_{\mathrm{M}}\right) x^{2}} d x
$$

with

$$
\begin{aligned}
\phi(x)=\alpha & K B_{o} e^{\frac{B_{o} N_{o}}{P_{\mathrm{R}} \beta} x_{\mathrm{R}}} \Gamma\left(0, \frac{B_{o} N_{o}}{P_{\mathrm{R}} \beta} x^{\eta_{\mathrm{R}}}\right)+ \\
& (1-\alpha) K B_{o} \int_{0}^{\infty} \frac{e^{-\frac{B_{o} N_{o}}{P_{\mathrm{R}} \beta} x^{\eta_{\mathrm{R}}} \gamma} \mathcal{L}_{I_{\mathrm{M}, \nu}\left(\frac{1}{P_{\mathrm{R}} \beta} x^{\eta_{\mathrm{R}}} \gamma\right)}}{1+\gamma} d \gamma,
\end{aligned}
$$

where $\Gamma(\cdot, \cdot)$ is the upper incomplete gamma function [12, (8.350)], and $\mathcal{L}_{I_{\mathrm{M}, \nu}}(\cdot)$ is given in (15).

Proof: Based on (1), $\bar{R}_{\mathrm{RRH}}$ is derived as

$$
\begin{aligned}
\bar{R}_{\mathrm{RRH}}= & \alpha K B_{o} \underbrace{\mathrm{E}\left\{\log _{2}\left(1+\gamma_{\mathrm{R}, k}\right)\right\}}_{\Xi_{1}} \\
& +(1-\alpha) K B_{o} \underbrace{\mathrm{E}\left\{\log _{2}\left(1+\gamma_{\mathrm{R}, \nu}\right)\right\}}_{\Xi_{2}} .
\end{aligned}
$$

In (11), $\Xi_{1}$ is calculated as

$$
\begin{aligned}
\Xi_{1} & =\int_{0}^{\infty} \mathrm{E}_{h_{\mathrm{R}, k}}\left\{\log _{2}\left(1+\frac{P_{\mathrm{R}} \beta}{B_{o} N_{o}} h_{\mathrm{R}, k} x^{-\eta_{\mathrm{R}}}\right)\right\} f_{\left|X_{o, \mathrm{R}}\right|}(x) \\
& =\frac{1}{\ln 2} \int_{0}^{\infty}\left\{\int_{0}^{\infty} \frac{1}{1+t} e^{-\frac{B_{o} N_{o}}{P_{\mathrm{R}} \beta} x^{\eta_{\mathrm{R}}} t} d t\right\} f_{\left|X_{o, \mathrm{R}}\right|}(x) d x \\
& =\frac{1}{\ln 2} \int_{0}^{\infty} e^{\frac{B_{o} N_{o}}{P_{\mathrm{R}} \beta} x^{\eta_{\mathrm{R}}}} \Gamma\left(0, \frac{B_{o} N_{o}}{P_{\mathrm{R}} \beta} x^{\eta_{\mathrm{R}}}\right) f_{\left|X_{o, \mathrm{R}}\right|}(x) d x,
\end{aligned}
$$

where $f_{\left|X_{o, \mathrm{R}}\right|}(x)$ is the probability density function (PDF) of the distance between the typical RRH and its intended user, using the similar approach in [13], $f_{\left|X_{o, \mathrm{R}}\right|}(x)$ is given by

$$
f_{\left|X_{o, \mathrm{R}}\right|}(x)=\frac{2 \pi \lambda_{\mathrm{R}}}{\mathcal{A}_{\mathrm{R}}} x e^{-\pi\left(\lambda_{\mathrm{R}}+\lambda_{\mathrm{M}}\right) x^{2}},
$$

where $\mathcal{A}_{\mathrm{R}}=\frac{\lambda_{\mathrm{R}}}{\lambda_{\mathrm{R}}+\lambda_{\mathrm{M}}}$ is the probability that a user is associated with the RRH.

For $\Xi_{2}$, we first provide the complementary cumulative distribution function (CCDF) of $\gamma_{\mathrm{R}, \nu}$ given a distance $\left|X_{o, \mathrm{R}}\right|=$ $x$, which is calculated as

$$
\begin{aligned}
& \bar{F}_{\gamma_{\mathrm{R}, \nu} \mid\left\{\left|X_{o, \mathrm{R}}\right|=x\right\}}(\gamma)=\operatorname{Pr}\left(\frac{P_{\mathrm{R}} h_{\mathrm{R}, \nu} \beta x^{-\eta_{\mathrm{R}}}}{I_{\mathrm{M}, \nu}+B_{o} N_{o}}>\gamma\right) \\
& =e^{-\frac{B_{o} N_{o}}{P_{\mathrm{R}} \beta} x^{\eta_{\mathrm{R}}} \gamma} \mathrm{E}_{\Phi_{\mathrm{M}}}\left\{e^{-\frac{1}{P_{\mathrm{R}} \beta} x_{\mathrm{R}} \gamma I_{\mathrm{M}, \nu}}\right\} \\
& =e^{-\frac{B_{o} N_{o}}{P_{\mathrm{R}} \beta} x^{\eta_{\mathrm{R}}} \gamma} \mathcal{L}_{I_{\mathrm{M}, \nu}}\left(\frac{1}{P_{\mathrm{R}} \beta} x^{\eta_{\mathrm{R}}} \gamma\right)
\end{aligned}
$$

where $\mathcal{L}_{I_{\mathrm{M}, \nu}}(\cdot)$ is the laplace transform of the PDF of $I_{\mathrm{M}, \nu}$, and is given by

$$
\begin{aligned}
& \mathcal{L}_{I_{\mathrm{M}, \nu}}(s)=\mathrm{E}\left\{\exp \left\{-\left(\sum_{\ell \in \Phi_{\mathrm{M}}} \frac{P_{\mathrm{M}}}{S} h_{\ell, \nu} \beta\left|X_{\ell, \mathrm{M}}\right|^{-\eta_{\mathrm{M}}}\right) s\right\}\right\} \\
& \stackrel{(a)}{=} \exp \left\{-\int_{x}^{\infty}\left(1-\frac{1}{\left(1+s \frac{P_{\mathrm{M}}}{S} \beta r^{-\eta_{\mathrm{M}}}\right)^{S}}\right) \lambda_{\mathrm{M}} 2 \pi r d r\right\} \\
& =\exp \left\{-\lambda_{\mathrm{M}} 2 \pi \sum_{\mu=1}^{S}\left(\begin{array}{c}
S \\
\mu
\end{array}\right)\left(s \frac{P_{\mathrm{M}}}{S} \beta\right)^{\mu} \frac{\left(-s \frac{P_{\mathrm{M}}}{S} \beta\right)^{-\mu+\frac{2}{\eta_{\mathrm{M}}}}}{\eta_{\mathrm{M}}}\right. \\
& \left.B_{\left(-s \frac{P_{\mathrm{M}}}{S} \beta x^{-\eta_{\mathrm{M}}}\right)}\left[\mu-\frac{2}{\eta_{\mathrm{M}}}, 1-S\right]\right\},
\end{aligned}
$$

where (a) is obtained by using the generating functional of PPP [14], $\mathrm{B}_{(\cdot)}[\cdot, \cdot]$ is the incomplete beta function [12, (8.391)]. Accordingly, $\Xi_{2}$ is given by

$$
\Xi_{2}=\frac{1}{\ln 2} \int_{0}^{\infty}\left[\int_{0}^{\infty} \frac{\bar{F}_{\gamma_{\mathrm{R}, \nu} \mid\left\{\left|X_{o, \mathrm{R}}\right|=x\right\}}(\gamma)}{1+\gamma} d \gamma\right] f_{\left|X_{o, \mathrm{R}}\right|}(x) d x .
$$

Substituting (12) and (16) into (11), we obtain (9).

Based on Theorem 1, the throughput for the RRH tier is given by

$$
\mathcal{T}_{\mathrm{R}}=\lambda_{\mathrm{R}} \bar{R}_{\mathrm{RRH}} .
$$

We next derive the throughput for a typical MBS, which can be written in a general-form following the approach in [15], however, using this approach will lead to intractable solution $x) d x$ in this work. As such, we present a tractable and closed-form lower bound expression.

Theorem 2. The throughput for a typical MBS can be lower bounded as the following closed-form expression.

$$
\bar{R}_{\mathrm{MBS}}^{\mathrm{L}}=(1-\alpha) K B_{o} S \log _{2}\left(1+e^{Z_{1}+\bar{Z}_{2}}\right),
$$


where

$$
\begin{aligned}
Z_{1}= & \ln \left(\frac{P_{\mathrm{M}}}{S} \beta\right)+\psi\left(N_{\mathrm{M}}-S+1\right) \\
& -\frac{\eta_{\mathrm{M}}}{2}\left(\psi(1)-\ln \left(\pi\left(\lambda_{\mathrm{R}}+\lambda_{\mathrm{M}}\right)\right)\right),
\end{aligned}
$$

and

$$
\bar{Z}_{2}=-\ln \left(\frac{P_{\mathrm{R}} \beta 2 \pi \lambda_{\mathrm{R}} \Gamma\left(2-\frac{\eta_{\mathrm{R}}}{2}\right)}{\left(\eta_{\mathrm{R}}-2\right)\left(\pi \lambda_{\mathrm{R}}+\pi \lambda_{\mathrm{M}}\right)^{1-\frac{\eta_{\mathrm{R}}}{2}}}+B_{o} N_{1}\right),
$$

respectively, where $\psi(\cdot)$ is the digamma function [16].

Proof: Based on (4), the throughput of a typical MBS is written as

$$
\bar{R}_{\mathrm{MBS}}=(1-\alpha) K B_{o} S \underbrace{\mathrm{E}\left\{\log _{2}\left(1+\gamma_{\mathrm{M}, \nu}\right)\right\}}_{\Xi_{3}},
$$

By using Jensen's inequality, a lower bound for $\Xi_{3}$ is given by

$$
\Xi_{3}^{\mathrm{L}}=\log _{2}\left(1+e^{Z_{1}+Z_{2}}\right)
$$

where

$$
Z_{1}=\mathrm{E}\left\{\ln \left(\frac{P_{\mathrm{M}}}{S} g_{\mathrm{M}, \nu} \beta\left|X_{o, \mathrm{M}}\right|^{-\eta_{\mathrm{M}}}\right)\right\},
$$

and

$$
Z_{2}=\mathrm{E}\left\{\ln \left(\frac{1}{I_{\mathrm{R}, \nu}+B_{o} N_{1}}\right)\right\} .
$$

We first calculate $Z_{1}$ as

$$
Z_{1}=\ln \left(\frac{P_{\mathrm{M}}}{S} \beta\right)+\mathrm{E}\left\{\ln \left(g_{\mathrm{M}, \nu}\right)\right\}-\eta_{\mathrm{M}} \mathrm{E}\left\{\ln \left(\left|X_{o, \mathrm{M}}\right|\right)\right\},
$$

Considering that $g_{\mathrm{M}, \nu} \sim \Gamma\left(N_{\mathrm{M}}-S+1,1\right), \mathrm{E}\left\{\ln \left(g_{\mathrm{M}, \nu}\right)\right\}$ is given by

$$
\begin{aligned}
\mathrm{E}\left\{\ln \left(g_{\mathrm{M}, \nu}\right)\right\} & =\int_{0}^{\infty} \frac{x^{N_{\mathrm{M}}-S} e^{-x}}{\left(N_{\mathrm{M}}-S\right) !} \ln (x) d x \\
& \stackrel{(b)}{=} \psi\left(N_{\mathrm{M}}-S+1\right),
\end{aligned}
$$

where (b) results from using $\int_{0}^{\infty} x^{v-1} e^{-\mu x} \ln x d x=$ $\mu^{-v} \Gamma(v)(\psi(v)-\ln \mu) \quad[12, \quad(4.352 .1)], \quad$ and for large $N_{\mathrm{M}}, \psi\left(N_{\mathrm{M}}-S+1\right) \approx \ln \left(N_{\mathrm{M}}-S+1\right)$ [17]. Then, $\mathrm{E}\left\{\ln \left(\left|X_{o, \mathrm{M}}\right|\right)\right\}$ is derived as

$$
\begin{aligned}
\mathrm{E}\left\{\ln \left(\left|X_{o, \mathrm{M}}\right|\right)\right\} & \stackrel{(c)}{=} \int_{0}^{\infty} \ln (x) f_{\left|X_{o, \mathrm{M}}\right|}(x) d x \\
& =\int_{0}^{\infty} \ln (x) \frac{2 \pi \lambda_{\mathrm{M}}}{\mathcal{A}_{\mathrm{M}}} x e^{-\pi\left(\lambda_{\mathrm{R}}+\lambda_{\mathrm{M}}\right) x^{2}} d x \\
& =\frac{1}{2}\left(\psi(1)-\ln \left(\pi\left(\lambda_{\mathrm{R}}+\lambda_{\mathrm{M}}\right)\right)\right) .
\end{aligned}
$$

In the step (c), $f_{\left|X_{o, M}\right|}(x)$ is the PDF of the distance between the typical MBS and its intended user, which can be directly obtained following (13), and $\mathcal{A}_{\mathrm{M}}=\frac{\lambda_{\mathrm{M}}}{\lambda_{\mathrm{R}}+\lambda_{\mathrm{M}}}$ is the probability that a user is associated with the MBS. By substituting (26) and (27) into (25), we obtain $Z_{1}$ as (19).
From (24), considering the convexity of $\log _{2}\left(\frac{1}{1+x}\right)$ and using Jensen's inequality, we derive the lower bound on the $Z_{2}$ as

$$
Z_{2} \geq \bar{Z}_{2}=\ln \left(\frac{1}{\mathrm{E}\left\{I_{\mathrm{R}, \nu}\right\}+B_{o} N_{1}}\right) .
$$

Then, we have

$$
\begin{aligned}
& \mathrm{E}\left\{I_{\mathrm{R}, \nu}\right\}=\int_{0}^{\infty} \mathrm{E}\left\{\sum_{j \in \Phi_{\mathrm{R}}} P_{\mathrm{R}} g_{j, \nu} \beta\left|X_{j, \mathrm{R}}\right|^{-\eta_{\mathrm{R}}}\right\} f_{\left|X_{o, \mathrm{M}}\right|}(x) d x \\
& \stackrel{(d)}{=} \int_{0}^{\infty}\left(P_{\mathrm{R}} \beta 2 \pi \lambda_{\mathrm{R}} \int_{x}^{\infty} r^{1-\eta_{\mathrm{R}}} d r\right) f_{\left|X_{o, \mathrm{M}}\right|}(x) d x \\
& =\int_{0}^{\infty} \frac{P_{\mathrm{R}} \beta 2 \pi \lambda_{\mathrm{R}}}{\eta_{\mathrm{R}}-2} x^{2-\eta_{\mathrm{R}}} f_{\left|X_{o, \mathrm{M}}\right|}(x) d x \\
& =\frac{P_{\mathrm{R}} \beta 2 \pi \lambda_{\mathrm{R}} \Gamma\left(2-\frac{\eta_{\mathrm{R}}}{2}\right)}{\left(\eta_{\mathrm{R}}-2\right)\left(\pi \lambda_{\mathrm{R}}+\pi \lambda_{\mathrm{M}}\right)^{1-\frac{\eta_{\mathrm{R}}}{2}}},
\end{aligned}
$$

where (d) results from using Campbell's theorem [18]

Substituting (29) into (28), we obtain $\bar{Z}_{2}$ as (20).

By using Theorem 2, the throughput for the MBSs tier is lower bounded as

$$
\mathcal{T}_{\mathrm{M}}^{\mathrm{L}}=\lambda_{\mathrm{M}} \bar{R}_{\mathrm{MBS}}^{\mathrm{L}}
$$

Thus, the overall throughput of the network is evaluated as

$$
\mathcal{T}_{\text {Net }}=\mathcal{T}_{\mathrm{R}}+\mathcal{T}_{\mathrm{M}}^{\mathrm{L}}=\lambda_{\mathrm{R}} \bar{R}_{\mathrm{RRH}}+\lambda_{\mathrm{M}} \bar{R}_{\mathrm{MBS}}^{\mathrm{L}} .
$$

\section{B. Energy Efficiency (EE)}

The EE for the RRHs tier is given by

$$
\mathrm{EE}_{\mathrm{R}}=\frac{\mathcal{T}_{\mathrm{R}}}{\lambda_{\mathrm{R}} P_{\mathrm{R}}^{\text {total }}}=\frac{\bar{R}_{\mathrm{RRH}}}{P_{\mathrm{R}}^{\text {total }}},
$$

where $\bar{R}_{\mathrm{RRH}}$ and $P_{\mathrm{R}}^{\text {total }}$ are given by (9) and (7), respectively. In the RRH tier, transmission over RBs that are only allocated to RRHs plays a dominant role in the overall throughput [8], compared to using RBs shared by the RRHs and MBSs. As a consequence, (32) can be approximately evaluated as

$$
\mathrm{EE}_{\mathrm{R}} \stackrel{(e)}{\approx} \frac{\alpha B_{o} \Xi_{1}}{\frac{P_{\mathrm{R}}}{\varepsilon_{\mathrm{R}}}}
$$

where (e) is obtained by omitting the power consumptions from static hardware and fronthaul link, compared to the RRH transmit power, and $\Xi_{1}$ is given by (12). It is implied from (33) that the EE for RRH transmission can be linearly improved by allocating more RBs to the RRHs. The EE for the MBSs tier is lower bounded as

$$
\mathrm{EE}_{\mathrm{M}}^{\mathrm{L}}=\frac{\mathcal{T}_{\mathrm{M}}^{\mathrm{L}}}{\lambda_{\mathrm{M}} P_{\mathrm{M}}^{\text {total }}}=\frac{\bar{R}_{\mathrm{MBS}}^{\mathrm{L}}}{P_{\mathrm{M}}^{\text {total }}},
$$

where $\bar{R}_{\mathrm{MBS}}^{\mathrm{L}}$ and $P_{\mathrm{M}}^{\text {total }}$ are given by (18) and (8), respectively. Lastly, the EE of the massive MIMO enabled heterogeneous $\mathrm{C}-\mathrm{RAN}$ is calculated as

$$
\begin{aligned}
\mathrm{EE}_{\mathrm{Net}} & =\frac{\text { Area throughput of the network }}{\text { Area Power Consumption of the network }} \\
& =\frac{\lambda_{\mathrm{R}} \bar{R}_{\mathrm{RRH}}+\lambda_{\mathrm{M}} \bar{R}_{\mathrm{MBS}}^{\mathrm{L}}}{\lambda_{\mathrm{R}} P_{\mathrm{R}}^{\text {total }}+\lambda_{\mathrm{M}} P_{\mathrm{M}}^{\text {total }}} .
\end{aligned}
$$




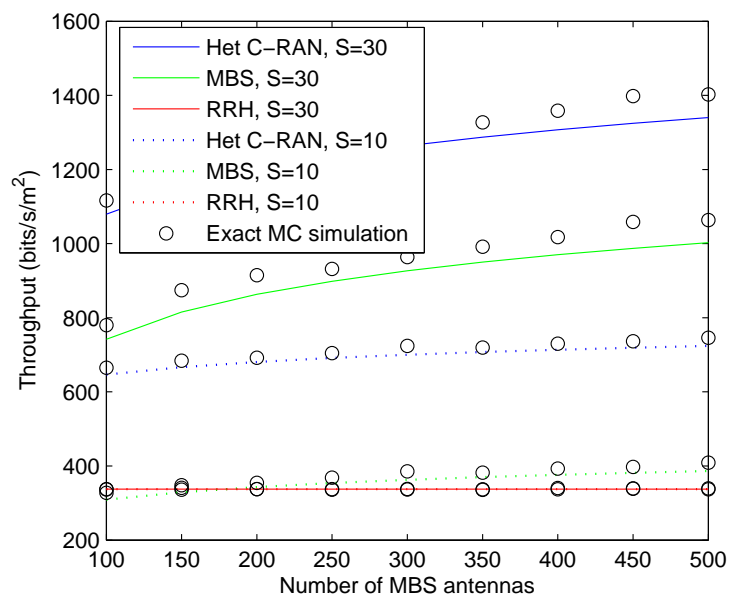

(a) Throughput.

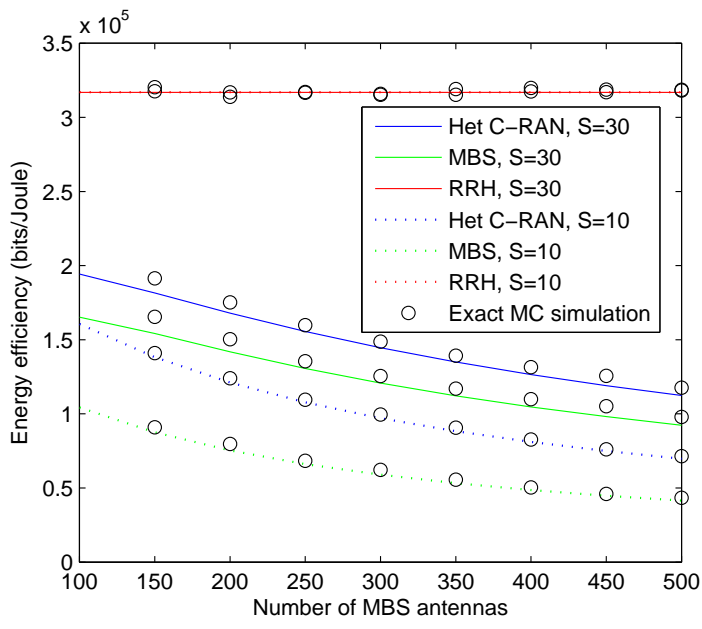

(b) Energy efficiency.

Fig. 2. Throughput and energy efficiency versus number of MBS antennas for different $S$.

\section{SIMULATION RESULTS}

In this section, we present numerical results to evaluate the throughput and EE in the massive MIMO enabled heterogeneous C-RAN (Het C-RAN). The density of MBSs is $\lambda_{\mathrm{M}}=\left(500^{2} \times \pi\right)^{-1} \mathrm{~m}^{-2}$ in a circular region with radius $1 \times 10^{4} \mathrm{~m}$. Such a network is assumed to operate at a carrier frequency of $1 \mathrm{GHz}$, the path loss exponents are $\eta_{\mathrm{M}}=3.0$ and $\eta_{\mathrm{R}}=3.6$, the MBS transmit power is $P_{\mathrm{M}}=40 \mathrm{dBm}$, the RRH transmit power is $P_{\mathrm{R}}=30 \mathrm{dBm}$, each RB bandwidth is $\mathrm{B}_{o}=200 \mathrm{KHz}$, and the total number of RBs is $K=25$. The noise power spectrum densities are $N_{0}=N_{1}=-162$ $\mathrm{dBm}$ [3]. The static hardware power consumption for RRH and $\mathrm{HPN}$ are $P_{\mathrm{R}}^{0}=0.1 \mathrm{~W}$ and $P_{\mathrm{M}}^{0}=10 \mathrm{~W}$, respectively, and the power consumption of the fronthaul link and backhaul link are $P_{\mathrm{fh}}=P_{\mathrm{bh}}=0.2 \mathrm{~W}$. We set the coefficients for efficiency of power amplifier $\varepsilon_{\mathrm{R}}=\varepsilon_{\mathrm{M}}=0.38$ and power consumption under LZFBF precoding in (8) as $\Delta_{1}=4.8$, $\Delta_{2}=0, \Delta_{3}=2.08 \times 10^{-8}, \Lambda_{1}=1, \Lambda_{2}=9.5 \times 10^{-8}$ and $\Lambda_{3}=6.25 \times 10^{-8}$ [11].

In the figures, Monte Carlo (MC) simulated exact values of the SE and EE marked by 'o' are numerically obtained to validate the analytical, and the green, red and blue curves represent the throughput and $\mathrm{EE}$ achieved by the MBSs tier, RRHs tier, and Het C-RAN, respectively. The throughput curves for the RRHs tier, MBSs tier and Het C-RAN are obtained from (17), (30), and (31), respectively. The EE curves for the RRHs tier, MBSs tier and Het C-RAN are obtained from (32), (34), and (35), respectively.

\section{A. The effects of Massive MIMO}

Fig. 2 shows the throughput and EE versus number of MBS antennas for different $S$. We set the density of RRHs as $\lambda_{\mathrm{R}}=$ $10 \times \lambda_{\mathrm{M}}$ and the S-FFR factor $\alpha=0.5$.

In Fig. 2(a), we see that the analytical throughput expression for RRHs tier has a good match with MC simulation, and the derived lower bounds can well predict the exact ones. The throughput of the MBSs tier and Het C-RAN increase with the number of MBS antennas, due to the increasing array gains. Moreover, serving more number of users in the massive MIMO macrocell can significantly improve the throughput of the MBSs tier and Het C-RAN, because of achieving more multiplexing gains. In addition, increasing the number of MBS antennas has negligible effect on the throughput of RRHs tier.

In Fig. 2(b), we see that RRHs tier achieves higher EE than the MBSs tier. EE of the MBSs tier and Het C-RAN decreases with increasing the number of MBS antennas, due to more power consumption from the precoding. However, serving more users in the macrocell can significantly improve EE, due to higher throughput as shown in Fig. 2(a). Again, increasing the number of MBS antennas has negligible effect on the EE of RRHs tier.

\section{B. The effects of S-FFR and RRHs tier density}

Fig. 3 shows the throughput and EE versus S-FFR factor for different RRH tier density. We set the number of MBS antennas as 200 and $S=15$.

In Fig. 3(a), we see that the throughput of RRHs tier increases with the S-FFR factor $\alpha$. When the density of RRHs tier is not dense (e.g. $\lambda_{\mathrm{R}}=10 \lambda_{\mathrm{M}}$ in this figure), the throughput of MBSs tier and Het C-RAN decrease with $\alpha$. The reason is that the MBSs tier plays a crucial role in the throughput of the entire network, and increasing $\alpha$ reduces the amount of RBs allocated to the MBSs tier. There is a critical point, exceeding which, the throughput achieved by the RRHs tier is higher than that in MBSs tier. In contrast, when the density of RRHs is dense (e.g. $\lambda_{\mathrm{R}}=30 \lambda_{\mathrm{M}}$ ), the throughput of the Het C-RAN increases with $\alpha$, which can be explained by the fact that the RRHs tier plays a key role in this case, and more number of RBs are allocated to the RRHs tier.

In Fig. 3(b), we see that RRHs tier achieves higher EE than the MBSs tier, and the EE of the RRHs tier and Het C-RAN increases with the S-FFR factor $\alpha$, given the RRHs tier density. 


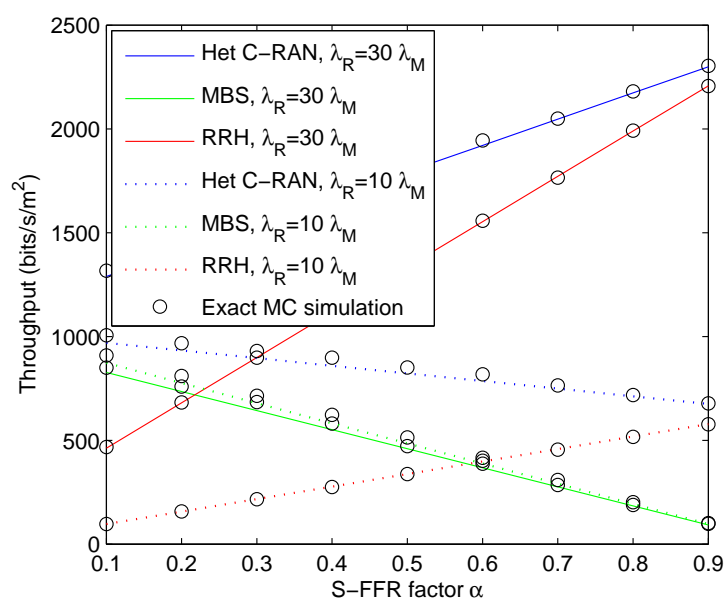

(a) Throughput.

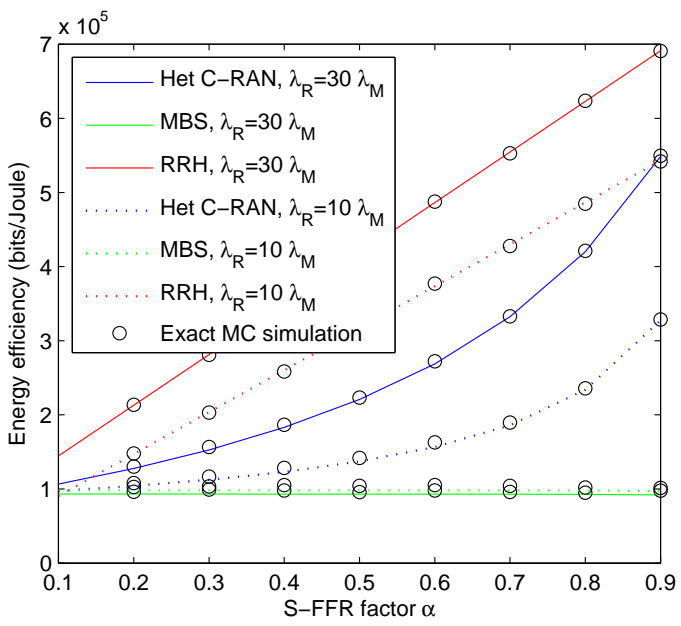

(b) Energy efficiency.

Fig. 3. Throughput and energy efficiency versus S-FFR factor for different RRH tier density.

When deploying more RRHs in the network, EE of the Het C-RAN significantly improves, due to the linear increase in the EE of the RRHs tier, as suggested in subsection III-B. There is an interesting phenomenon that the S-FFR factor has negligible impact on the EE of the MBSs tier. The reason is that for massive MIMO empowered MBS, the low power cost for backhaul link and static hardware can be omitted compared with the massive MIMO precoding, in this condition, each RB of the MBS has the same EE, which means it is independent of the frequency resource allocation.

\section{CONCLUSiOnS}

In this paper, we introduced the two-tier massive MIMO aided heterogeneous C-RAN consisting of massive MIMO macrocell base stations as the first tier and RRHs as the second tier. In such networks, the implementation of the soft fractional frequency reuse (S-FFR) was utilized to mitigate the inter-tier interference. We first obtained the exact expressions for the throughput of the RRHs tier. Then, we presented a tractable approximation approach for evaluating the throughput of the macrocell base stations tier. Numerical results collaborated our analysis and showed that massive MIMO with dense deployment of RRHs can significantly enhance the throughput of heterogeneous C-RAN. More frequency resources allocated to the RRHs improves the network EE. The S-FFR factor should be carefully chosen, since its effect depends on the density of the RRHs.

\section{REFERENCES}

[1] A. Checko, H. Christiansen, Y. Yan, L. Scolari, G. Kardaras, M. Berger, and L. Dittmann, "Cloud ran for mobile networks-a technology overview," IEEE Commun. Surveys \& Tutorials, vol. 17, no. 1, pp. 405426, 2015.

[2] E. Hossain and M. Hasan, "5G cellular: Key enabling technologies and research challenges," IEEE Instrumentation Measurement Mag., vol. 18, no. 3, pp. 11-21, Jun. 2015.

[3] M. Peng, C. Wang, V. Lau, and H. Poor, "Fronthaul-constrained cloud radio access networks: insights and challenges," IEEE Wireless Commun., vol. 22, no. 2, pp. 152-160, 2015.
[4] Z. Ding and H. Poor, "The use of spatially random base stations in cloud radio access networks," IEEE Signal Process. Lett., vol. 20, no. 11, pp. 1138-1141, Nov. 2013.

[5] S. Zaidi, A. Imran, D. C. Mclernon, and M. Ghogho, "Characterizing coverage and downlink throughput of cloud empowered hetnets," IEEE Commun. Lett., vol. 19, no. 6, pp. 1013-1016, 2015.

[6] F. Khan, H. He, J. Xue, and T. Ratnarajah, "Performance analysis of cloud radio access networks with distributed multiple antenna remote radio heads," IEEE Trans. Signal Process., vol. 63, no. 18, pp. 47844799, Sep. 2015.

[7] M. Peng, Y. Li, J. Jiang, J. Li, and C. Wang, "Heterogeneous cloud radio access networks: a new perspective for enhancing spectral and energy efficiencies," IEEE Wireless Commun., vol. 21, no. 6, pp. 126-135, 2014.

[8] M. Peng, K. Zhang, J. Jiang, J. Wang, and W. Wang, "Energy-efficient resource assignment and power allocation in heterogeneous cloud radio access networks," IEEE Trans. Veh. Technol., pp. 1-13, 2015.

[9] H. Q. Ngo, E. G. Larsson, and T. L. Marzetta, "Energy and spectral efficiency of very large multiuser MIMO systems," IEEE Trans. Commun., vol. 61, no. 4, pp. 1436-1449, Apr. 2013.

[10] K. Hosseini, W. Yu, and R. S. Adve, "Large-scale MIMO versus network MIMO for multicell interference mitigation," IEEE J. Sel. Areas Commun., vol. 8, no. 5, pp. 930-941, Oct. 2014.

[11] E. Björnson, L. Sanguinetti, J. Hoydis, and M. Debbah, "Designing multi-user MIMO for energy efficiency: When is massive MIMO the answer?" in Proc. 2014 IEEE WCNC, Apr. 2014.

[12] I. S. Gradshteyn and I. M. Ryzhik, Table of Integrals, Series and Products, 7th ed. San Diego, C.A.: Academic Press, 2007.

[13] H.-S. Jo, Y. J. Sang, P. Xia, and J. G. Andrews, "Heterogeneous cellular networks with flexible cell association: A comprehensive downlink SINR analysis," IEEE Trans. Wireless Commun., vol. 11, no. 10, pp. 34843495, Oct. 2012.

[14] M. Haenggi, Stochastic Geometry for Wireless Networks. Cambridge University Press, 2013.

[15] M. Di Renzo and P. Guan, "Stochastic geometry modeling of coverage and rate of cellular networks using the Gil-Pelaez inversion theorem," IEEE Commun. Lett., vol. 18, no. 9, pp. 1575-1578, 2014.

[16] M. Abramowitz and I. A. Stegun, Handbook of Mathematical Functions with Formulas, Graphs, and Mathematical Tables, 9th ed. New York: Dover Publications, 1970.

[17] L. Wang, H. Q. Ngo, M. Elkashlan, T. Q. Duong, and Kai-Kit Wong, "Massive MIMO in spectrum sharing networks: Achievable rate and power efficiency," IEEE Systems Journal, 1-12, 2015.

[18] F. Baccelli and B. Błszczyszyn, Stochastic Geometry and Wireless Networks, Volume I: Theory. Now Publishers Inc. Hanover, MA, USA, 2009. 